\title{
Origins of the Failure of Classical Nucleation Theory for Nanocellular Polymer Foams
}

\author{
Yeongyoon Kim, ${ }^{1}$ Chul B. Park, ${ }^{2}$ P. Chen,${ }^{3,4}$ and Russell B. Thompson ${ }^{1,4, *}$ \\ ${ }^{1}$ Department of Physics and Astronomy, \\ University of Waterloo, 200 University Avenue West, \\ Waterloo, Ontario, Canada N2L $3 G 1$ \\ ${ }^{2}$ Microcellular Plastics Manufacturing Laboratory, \\ Department of Mechanical and Industrial Engineering, \\ University of Toronto, 5 King's College Road, \\ Toronto, Ontario, Canada M5S $3 G 8$ \\ ${ }^{3}$ Department of Chemical Engineering, \\ University of Waterloo, 200 University Avenue West, \\ Waterloo, Ontario, Canada N2L 3G1 \\ 4 Waterloo Institute for Nanotechnology, \\ University of Waterloo, 200 University Avenue West, \\ Waterloo, Ontario, Canada N2L $3 G 1$
}

This document is the accepted manuscript version of a published article. Published by The Royal Society of Chemistry in the journal "Soft Matter" issue 16, DOI: 10.1039/C1SM05575E 


\begin{abstract}
Relative nucleation rates for fluid bubbles of nanometre dimensions in polymer matrices are calculated using both classical nucleation theory and self-consistent field theory. An identical model is used for both calculations showing that classical nucleation theory predictions are off by many orders of magnitude. The main cause of the failure of classical nucleation theory can be traced to its representation of a bubble surface as a flat interface. For nanoscopic bubbles, the curvature of the bubble surface is comparable to the size of the polymer molecules. Polymers on the outside of a curved bubble surface can explore more conformations than can polymers next to a flat interface. This reduces the free energy of the curved interface which leads to a significantly smaller barrier energy to nucleation and thus a much higher nucleation rate. Also, there is a reduction of unfavorable energetic contacts between polymer and fluid molecules in the vicinity of a curved interface. Polymers on the outside of a curved interface are less likely to find a portion of themselves in the interior of the unfavorable fluid bubble. A secondary cause of the failure of classical nucleation theory is due to the collapse of the bulk region inside the bubble. As the radius of a bubble is reduced, eventually the diffuse walls collide causing increased mixing of polymer and fluid molecules everywhere. This causes a reduction of internal energy associated with the interface, leading to smaller nucleation barrier energies and, again, a reduced barrier energy to nucleation.
\end{abstract}

PACS numbers: 82.70.Rr, 47.57.Bc, 68.03.Cd, 36.20.Fz, 33.15.Bh, 36.20.Ey, 64.75.Jk

${ }^{*}$ Electronic address: thompson@uwaterloo.ca 


\section{INTRODUCTION}

Polymer foams, which consist of polymer matrices with fluid bubble inclusions, are used in furniture, automotive parts, packaging, construction materials, insulation, and many other areas [1]. A high quality foam should have a large number of small and uniform cells (bubbles). This requires cell nucleation to take place rapidly when creating the foam. Classical nucleation theory (CNT) is often used to predict bubble nucleation rates [2-6] so that one can engineer polymer foams with smaller cell sizes - microcellular foams, for example. Further improvements are being sought by reducing cell sizes to nanometre dimensions to create nanocellular foams [2]. When working at the nanoscale however, the science and engineering changes fundamentally. In particular, issues arise with the use of CNT as some assumptions break down for nano-sized bubbles. Oxtoby has reviewed CNT assumptions [7]: 1. CNT assumes an energy barrier to nucleation always exists, which is not always the case. 2. CNT assumes a bubble always has bulk properties, even it if is very small, and that it always has a sharp interface. 3. CNT represents a bubble interface by an infinite flat planar surface. In addition to these limitations listed by Oxtoby, CNT contains no kinetic information. This can be very important in polymer foams, for example in nonstationary diffusion growth in nanosized pores [8].

Self-consistent field theory (SCFT) is free from the limitations listed by Oxtoby. SCFT is a mean field, statistical mechanical theory well suited to studying inhomogenous polymer systems $[9,10]$ including interfaces in polymer foams [11-14]. In this paper, we calculate critical radii, nucleation barriers and relative nucleation rates in polymer bubble systems using SCFT and compare to results found using CNT for the same systems. Although SCFT is like CNT in that it doesn't contain any kinetic information, this is actually advantageous in that a comparison between the two theories is on an equal footing - more will be said about the kinetic limitations of SCFT in the theory section. CNT requires certain inputs (surface tension and volume free energy density) which we compute using SCFT. We have therefore an ideal system that, by construction, would give perfect agreement between SCFT and CNT if CNT was completely correct. Although our model system is not expected to be quantitative, it shows correct qualitative features and is free of confounding factors in judging CNT. Furthermore, the identical model used means all disagreements found are systemic to CNT and independent of quantitative arguments. SCFT uses a coarse-grained 
microscopic model which (unlike other approaches to polymer bubble nucleation $[15,16]$ ) includes molecular degrees of freedom, and so by comparing SCFT to the same model using $\mathrm{CNT}$, the microscopic origins of the failure of CNT are unambiguously revealed. Specifically, we find that the increased conformations available to polymer molecules adjacent to a curved cell interface compared to a flat planar interface moderates the free energy difference between small bubbles and large bubbles. The curved interface also allows more space for polymers to avoid intruding into the chemically unfavorable bubble region compared to a flat interface. Taken together, the increased conformations and reduced internal energy resulting from the existence of bubble curvature on the length scale of the polymer reduce significantly the nucleation barrier energy for nanocellular foams and changes nucleation rates by many orders of magnitude. A secondary cause of the failure of CNT, which is only important for very small bubbles, is due to the disappearance of the bulk region inside the bubble. This causes increased mixing of polymer and foaming agent both inside and outside the bubble and contributes to the deviation of SCFT and CNT predictions.

In section 2, we summarize CNT and give our SCFT model for a polymer bubble. Although heterogenous nucleation is a much more important factor determining nucleation rates in polymer foams than homogenous nucleation $[17,18]$, we will examine only the latter, following the philosophy that homogenous nucleation is an appropriate place to start when testing CNT predictions given that CNT, in its simplest form, is a theory for homogenous nucleation $[7,19,20]$. Furthermore, many heterogenous nucleation theories are modifications of homogenous nucleation quantities [17, 18], so corrections to the latter will affect the former. In section 3, we show an example of the differences between critical radii, nucleation barriers and nucleation rates predicted by SCFT and CNT. These results provide some circumstantial evidence that there may be much more homogenous nucleation taking place in polymers foams than previously thought and that heterogenous nucleation rate predictions may also be inaccurate. We discuss the microscopic origins of the differences between SCFT and CNT in this section. In section 4, we review our conclusions and list possible future directions of research. 


\section{THEORY}

Classical nucleation theory (CNT) assumes that phase transitions in fluids take place via nucleation and growth, with bubbles of the new phase nucleating in a matrix of the old phase at a rate $J$ given by

$$
J=J_{0} \exp \left(-\frac{\Delta F^{*}}{k_{B} T}\right)
$$

where $J_{0}$ is a prefactor associated with the characteristic time scales of motion in the system, $k_{B}$ is Boltzmann's constant and $T$ is the temperature $[1,7]$. The nucleation rate depends most importantly on the activation barrier $\Delta F^{*}$, which is the free energy of formation of a typical bubble of a radius $R$ equal to the critical radius $R^{*}$. The critical radius of a bubble is that radius smaller than which the bubble shrinks and disappears and larger than which the bubble grows continually. According to CNT, the free energy $\Delta F$ necessary to form a typical bubble of an arbitrary radius is

$$
\Delta F(R)=4 \pi R^{2} \gamma-\frac{4 \pi}{3} R^{3} \Delta \mathcal{F}_{V}
$$

The first term of on the right hand side of equation (2) is an energy penalty due to the existence of a bubble surface. $\gamma$ is the surface tension between the two phases assuming an infinite flat planar interface. The second term of (2) is an energy reduction due to the volume of the new phase bubble. We shall call $\Delta \mathcal{F}_{V}$ the volume free energy density and we will define it such that it has a positive value. The second term of CNT is more commonly written in terms of a pressure difference $\Delta p$ rather than $\Delta \mathcal{F}_{V}[1,19]$. The units are the same however and the latter offers a more general interpretation - we find it convenient to follow the presentation of Oxtoby [7] or Jones and Richards [20] in the use of $\Delta \mathcal{F}_{V}$. Maximization of equation (2) gives the critical radius $R^{*}$ and thus the activation barrier $\Delta F^{*}$.

In this article, script $\mathcal{F}$ will denote free energy densities, latin $F$ extensive free energies and tildes dimensionless quantities. A self-consistent field theory (SCFT) model for a bubble of fluid in a polymer matrix can be summarized by the free energy functional

$$
\begin{aligned}
\tilde{\mathcal{F}} \equiv \frac{N F}{\rho_{0} k_{B} T V}= & -\left(1-\phi_{s}\right) \ln \left[\frac{Q_{p}}{V\left(1-\phi_{s}\right)}\right]-\frac{\phi_{s}}{\alpha} \ln \left(\frac{Q_{s} \alpha}{V \phi_{s}}\right) \\
& +\frac{1}{V} \int d \mathbf{r}\left[\chi N \varphi_{p}(\mathbf{r}) \varphi_{s}(\mathbf{r})-w_{s}(\mathbf{r}) \varphi_{s}(\mathbf{r})-w_{p}(\mathbf{r}) \varphi_{p}(\mathbf{r})\right] .
\end{aligned}
$$

where $\varphi_{p}(\mathbf{r})$ and $\varphi_{s}(\mathbf{r})$ are the local (position dependent) volume fractions of polymer and solvent, respectively. The term "solvent" is often used in SCFT to refer to molecules lacking 
polymeric internal degees of freedom. We will use this term interchangeably with "fluid" to refer to the gas or fluid phase of a polymer foam system. In addition to equation (3), an incompressibility constraint, $\varphi_{p}(\mathbf{r})+\varphi_{s}(\mathbf{r})=1$, is enforced. The use of an incompressible equation of state is not realistic for a polymer foam system, but it has been found to show the correct qualitative behavior of the interface $[2,13,14]$ and it removes complications that can cloud interpretations. Realistic equation of state effects can be added to the formalism $[11,12]$ - see Conclusions and Outlook section 4. The total system volume fractions for polymer and solvent are $\left(1-\phi_{s}\right)$ and $\phi_{s}$, respectively, and single molecule partition functions are given by $Q_{p}$ and $Q_{s}$, respectively. The ratio of the volume of a solvent molecule to a polymer molecule is denoted by $\alpha$, with the volume of one polymer segment being $\rho_{0}^{-1}$ and the degree of polymerization being $N$. The segregation between solvent molecules and polymer segments is given by a Flory-Huggins parameter $\chi \cdot w(\mathbf{r})_{s}$ or $p$ are the mean fields felt by each solvent molecule or polymer segment due to interactions with all other molecules and segments in the system. The left hand side of $(3)$ is the system free energy $(F)$ per system volume $(V)$, made dimensionless by dividing by the thermal energy $k_{B} T$ and multiplying by the volume of one polymer, $N / \rho_{0}$. A set of coupled equations are derived from the free energy functional (3) when combined with the incompressibility constraint, with one equation for each of $\varphi_{p}(\mathbf{r}), \varphi_{s}(\mathbf{r}), w_{p}(\mathbf{r})$ and $w_{s}(\mathbf{r})$. These equations, together with incompressibility, are solved self-consistently and numerically using reflecting boundary conditions. Details of the SCFT approach, including its limitations such as the neglect of fluctuations, can be found in the review of Matsen [10], the book of Fredrickson [9] or, for polymer/solvent interface systems, the review of Binder et al. [11] and our previous work on the subject [12].

SCFT can be used to calculate the surface tension of an infinite flat planar solvent/polymer interface. The surface tension is the excess free energy of the interface divided by the interfacial area. The excess free energy density is found by subtracting the bulk free energy densities on either side of the interface (the phase separated free energy densities) from the system free energy density, equation (3):

$$
\tilde{\mathcal{F}}_{\mathrm{ex}}=\tilde{\mathcal{F}}-\tilde{\mathcal{F}}_{s}
$$

This is schematically summarized in figure 1, panels (a) and (b). Panel (a) shows a typical interface predicted by SCFT using formula (3). Panel (b) shows two, independent, homogenous systems with polymer and solvent fractions corresponding to the phase separated 


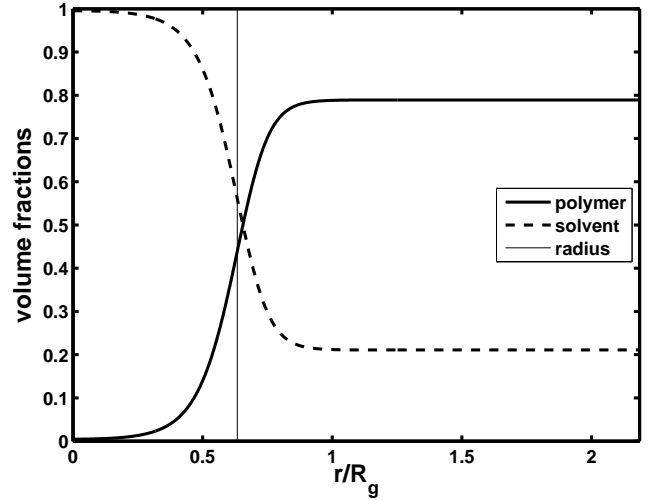

(a)

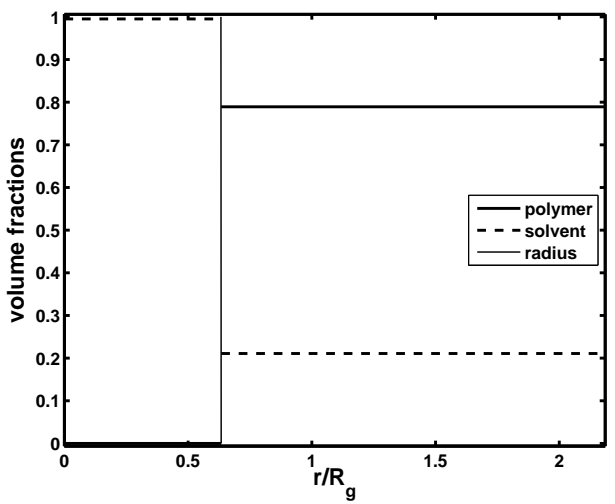

(b)

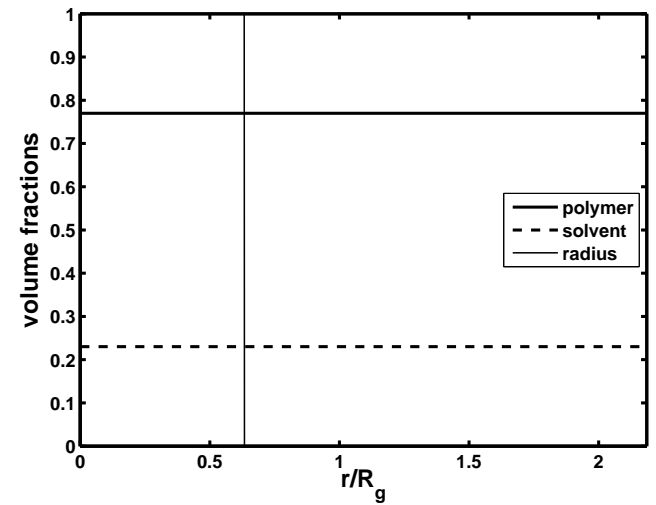

(c)

FIG. 1: Schematic showing the structures used to calculate various SCFT quantities for comparison with CNT. In all figures, the abscissa is a dimensionless radial coordinate and the ordinate shows volume fractions according to the legends. (a) A typical SCFT prediction of a polymer/fluid interface from equation (3). The vertical line is the location of the defined bubble radius. (b) Two, independent, homogenous systems with polymer and fluid volume fractions corresponding to the phase separated values on either side of the interface of panel (a). The vertical radius marker now divides the two sub-systems. (c) A homogenous, bubble free, structure with the same overall volume fractions as panel (a). The vertical radius marker is left in for comparison. To calculate the excess free energy density $\tilde{\mathcal{F}}_{\text {ex }}$, one takes the difference of free energy density between panels (a) and (b) (equation (4)). To calculate the volume free energy density $\widetilde{\Delta \mathcal{F}_{V}}$, one takes the difference of free energy density between panels (b) and (c) (equation (10)). To calculate the free energy density of formation of a bubble of radius $\tilde{R}$, one takes the difference of free energy density between panels (a) and (c) (equation (12)). 
values on either side of the interface of panel (a). To calculate the excess free energy density $\tilde{\mathcal{F}}_{\text {ex }}$, one essentially takes the difference of free energy density between panels (a) and (b) (equation (4)) as we will now describe. The total phase separated free energy density is given by $[10,12]$

$$
\tilde{\mathcal{F}}_{s}=\tilde{\mathcal{F}}_{h}^{(1)}\left(\frac{V_{1}}{V}\right)+\tilde{\mathcal{F}}_{h}^{(2)}\left(\frac{V_{2}}{V}\right)
$$

where $\tilde{\mathcal{F}}_{h}^{(1)}$ and $\tilde{\mathcal{F}}_{h}^{(2)}$ are the bulk homogenous free energy densities on either side of the interface, illustrated in figure 1(b). These are found using the homogenous version of the free energy functional (3) which is

$$
\tilde{\mathcal{F}}_{h} \equiv \frac{N F_{h}}{\rho_{0} k_{B} T V}=\left(1-\phi_{s}\right) \ln \left(1-\phi_{s}\right)+\frac{\phi_{s}}{\alpha} \ln \left(\frac{\phi_{s}}{\alpha}\right)+\chi N\left(1-\phi_{s}\right) \phi_{s}
$$

where the bulk volume fractions $\phi_{s}^{(1)}$ and $\phi_{s}^{(2)}$ on either side of the interface would be used in equation (6) to get $\tilde{\mathcal{F}}_{h}^{(1)}$ and $\tilde{\mathcal{F}}_{h}^{(2)}$, respectively. $V_{1}$ and $V_{2}$ are the volumes of the homogenous, phase separated regions given by

$$
V_{1}+V_{2}=V
$$

and

$$
\frac{V_{1}}{V}=\frac{\phi_{s}^{(2)}-\phi_{s}}{\phi_{s}^{(2)}-\phi_{s}^{(1)}}
$$

which conserves the total volume of solvent of the inhomogenous system (3) in the phase separated expression (5) - see references 10 and 12. Dividing the excess free energy by interfacial area $A$, the dimensionless surface tension $\tilde{\gamma}$ is [21]

$$
\tilde{\gamma} \equiv \frac{6 R_{g} \gamma}{a^{2} \rho_{0} k_{B} T}=\left(\frac{V}{R_{g}}\right) \frac{\tilde{\mathcal{F}}_{\text {ex }}}{A}
$$

where $a$ is the average length of a polymer segment and $R_{g}$ is the unperturbed radius of gyration of a polymer. $R_{g}$ will be used as the unit of length for all results presented. The multiplication by volume on the right hand side of equation (9) converts the (dimensionless) excess free energy density into an extensive free energy. More details on calculating surface tensions and excess free energies in SCFT can be found in references 10 and 12 .

A flat interface surface tension found from equation (9) can be used in CNT, but one also needs the volume free energy density $\Delta \mathcal{F}_{V}$. One can find this by taking the difference between the phase separated free energy density $\mathcal{F}_{s}$ given by equation (5) and the homogenous free energy density $\mathcal{F}_{h}$ given by equation (6). In equation (6), the total system volume 
fraction $\phi_{s}$ would be used.

$$
\widetilde{\Delta \mathcal{F}}_{V} \equiv \frac{N \Delta \mathcal{F}_{V}}{\rho_{0} k_{B} T}=\frac{V}{V_{1}}\left(\tilde{\mathcal{F}}_{h}-\tilde{\mathcal{F}}_{s}\right)
$$

This is illustrated schematically in figure 1 panels (b) and (c). Panel (b) shows two, independent, homogenous systems with polymer and solvent fractions corresponding to the phase separated values on either side of the interface of panel (a) while panel (c) shows a homogenous, bubble free, structure with the same overall volume fractions as panel (a). To calculate the volume free energy density $\widetilde{\Delta \mathcal{F}}_{V}$, one essentially takes the difference of free energy density between panels (b) and (c) (equation (10)). In (10), $\left(V_{1} / V\right)^{-1}$ is given by equation (8); this pre-factor is a convenience used to split the full free energy density (3) into surface and volume terms as will be explained later in this section. The SCFT surface tension (9) and volume free energy density (10) can be calculated for a flat, infinite planar surface and used in a dimensionless version of equation (2)

$$
\widetilde{\Delta F}(R) \equiv \frac{N \Delta F}{\rho_{0} k_{B} T R_{g}^{3}}=4 \pi\left(\frac{R}{R_{g}}\right)^{2} \tilde{\gamma}-\frac{4 \pi}{3}\left(\frac{R}{R_{g}}\right)^{3} \widetilde{\Delta \mathcal{F}_{V}}
$$

which gives the CNT prediction for the free energy to form a bubble as a function of bubble radius.

One can also calculate $\widetilde{\Delta F}$ directly from SCFT. One subtracts the homogenous free energy density (6) from the system free energy density (3) and multiplies by $V$ to get a (dimensionless) extensive free energy rather than a free energy density:

$$
\widetilde{\Delta F}=\frac{V}{R_{g}^{3}}\left(\tilde{\mathcal{F}}-\tilde{\mathcal{F}}_{h}\right)
$$

This is illustrated schematically in figure 1 panels (a) and (c). Panel (a) shows a typical interface predicted by SCFT from formula (3) while panel (c) shows a homogenous, bubble free, structure with the same overall volume fractions as panel (a). To calculate the free energy density of formation of a bubble of radius $\tilde{R}$, one essentially takes the difference of free energy density between panels (a) and (c) (equation (12)). From definitions (9) and (10), this can be rewritten in the suggestive form

$$
\widetilde{\Delta F}=\left(\frac{A}{R_{g}^{2}}\right) \tilde{\gamma}-\left(\frac{V_{1}}{R_{g}^{3}}\right) \widetilde{\Delta \mathcal{F}_{V}}
$$

This is just a geometry independent generalization of the CNT formula (11). It is for this reason that the factor $\left(V_{1} / V\right)^{-1}$ was included in the definition of (10) and it shows that 
$V_{1}$ is being defined as the volume of a bubble of surface area $A$. For spherical coordinates, equation (13) becomes identical to (11) but now it is no longer the case that the surface tension (9) and volume free energy (10) are evaluated for an infinite flat planar surface. We are now solving the full SCFT free energy functional for a spherical bubble symmetry with no CNT assumptions. Unlike the fixed flat interface surface tension, $\widetilde{\Delta F}(R)$ depends on the system volume $V$ used in the SCFT calculation (as does the volume free energy). To get an unambiguous value, one must use in formula (3) the volume $V$ that gives the prescribed total volume fraction $\phi_{s}$. That is, one must match to experimental conditions. For $\widetilde{\Delta F}(R)$ therefore, one must change the system volume and recalculate for each morphology of differing radius [22]. To associate each morphology with a radius $R$ one must define the radius of the structure one finds. In CNT, the radius is uniquely defined since the bubble interface is assumed to be a sharp step. When calculating a bubble morphology in SCFT one finds a more realistic diffuse interface. The location of this interface is therefore a matter of definition [23]. Since we are comparing with CNT, we use the CNT definition as given by equation (8). This means that $V_{1}$ is the volume of a spherical bubble, with the radius being derived from this volume. This radius corresponds to the dividing surface defined in figure 1, panel (b) and shown against the diffuse interface in panel (a). Since these two figures show systems with the same number of molecules, the radius is defined at the equimolecular surface. Therefore, surface tensions calculated using this radius will be consistent with thermodynamic definitions of surface tension and with excess free energies per area calculated in grand canonical formalisms. The reader should note that the SCFT approach differs from CNT in that it is thermodynamically consistent: the overall volume fraction $\phi_{s}$ is required as input, and the system volume must be changed for each radius of bubble in order to preserve this volume fraction. In this way, the single bubble being modeled is a representative one for an environment in which many bubbles are nucleating and which therefore changes as the bubbles grow. Similar issues have been discussed by Leung et al. from a different perspective [2, 24].

Since a nucleating bubble is unstable in that it will necessarily shrink or grow, an equilibrium statistical mechanical method like SCFT is not an obvious way to model such systems. Previous authors have worked in grand canonical formalisms and measured the critical radius by determining where computations become unstable [11, 19] or by introducing constraints on the morphology $[25,26]$. Oxtoby pointed out that bubbles are stable in canonical for- 
malisms where the free energies can be correctly evaluated [7]. We use a constraint-free, canonical ensemble here. The bubbles we study are stable against growth above the critical radius. At and below the critical radius, a homogenous solution is always found. For these smaller radii, intermediate accuracy calculations show the bubble is spontaneously shrinking during the course of the calculation, as expected.

There is also the related issue of whether real bubbles in polymer foams reach quasiequilibrium as modeled here with SCFT. While there will certainly be cases where this is not true, such as when the polymer is near its glass transition or in the late stages of phase separation where viscoelastic effects are more pronounced, there will also be many systems where the quasi-equilibrium picture should be valid [11]. In any case, CNT suffers the same kinetic limitations so, as mentioned in the introduction, SCFT is an appropriate tool to reveal the failure of CNT within such a non-kinetic picture.

\section{RESULTS AND DISCUSSION}

We examine systems with $\alpha=0.01$ ( $\alpha$ is related to polymer molecular weight) , $\chi N$ ranging from 120 to 160 ( $\chi N$ is related to temperature and chemistry) and $\phi_{s}$ ranging from 0.2 to 0.33 ( $\phi_{s}$ is the volume fraction of gas), always chosen such that we are working in the nucleation and growth regime. In what follows, we depict and discuss results for $\alpha=0.01$, $\chi N=140$ and $\phi_{s}=0.23$, but our conclusions are valid for the range of values we studied. Figure 2 shows example profiles for two different bubble sizes. One notices that the bulk volume fractions $\phi_{s}^{(1)}$ and $\phi_{s}^{(2)}$ are slightly different for the two profiles and it is found they are also slightly different from the infinite, flat planar values. The bulk volume fractions are therefore weak functions of the bubble radius. It is the radially dependent rather than the flat planar values of $\phi_{s}^{(1)}$ and $\phi_{s}^{(2)}$ that we use to calculate the spherical surface tension since these radially dependent values reflect the non-equilibrium conditions in which the bubble exists for any given radius.

Figure 3 shows the free energy of the existence of a bubble, $\widetilde{\Delta F}$, as a function of bubble radius $\tilde{R} \equiv R / R_{g}$. The solid line is the prediction of CNT (equation (11)) based on a surface tension value of $\tilde{\gamma}=3.3$ and a volume free energy density of $\widetilde{\Delta \mathcal{F}}_{V}=7.1$. These are the values predicted by SCFT based on a flat interface that reaches bulk conditions on

both sides of the interface. The open circles in figure 3 are the predictions for $\widetilde{\Delta F}$ directly 


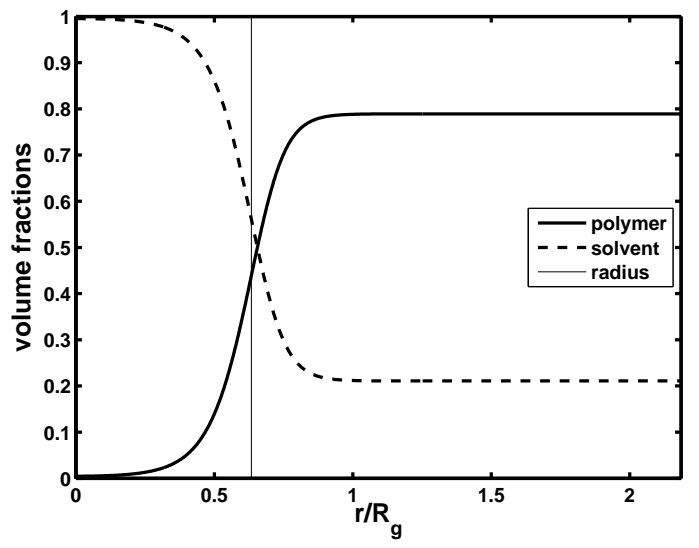

(a)

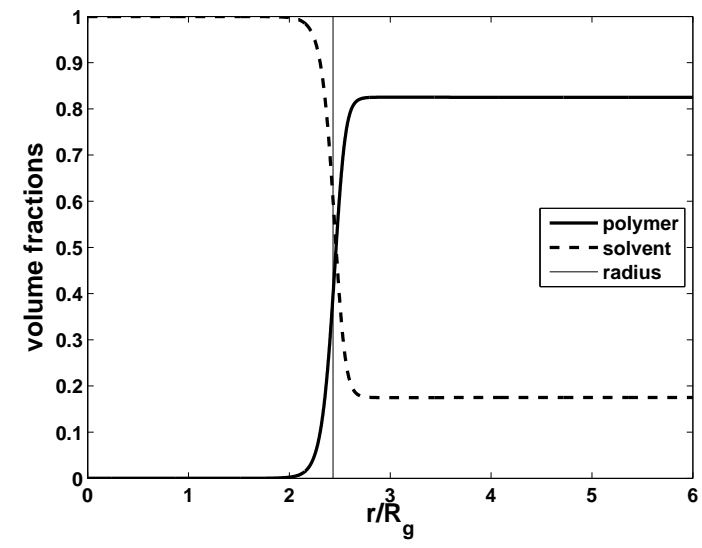

(b)

FIG. 2: Local volume fraction profiles for a bubble of solvent in a polymer matrix. The abscissa is a dimensionless radial coordinate and the ordinate shows volume fractions according to the legends. (a) Smaller bubble. (b). Larger bubble. In both panels, the definition of the location of a bubble interface, and therefore the radius of the bubble, is given by the vertical solid line.

from SCFT calculated from formula (13). The dashed line is a guide to the eye. We see that CNT overestimates the size of the critical radius by a factor of almost 1.5 and, more significantly, it overestimates the size of the energy barrier to nucleation by more than a factor of six. When used in the exponential nucleation rate formula (1), assuming the same prefactor for both CNT and SCFT, we find that CNT underestimates the nucleation rate by a factor of more than $5 \times 10^{5}$. Evans and Oxtoby have studied non-polymeric systems using classical density functional theory and they too find orders of magnitude deviations from CNT - see their figure 4 [19]. In polymer foam systems, heterogenous nucleation is thought to dominate bubble nucleation because CNT predicts such a small rate for homogenous nucleation $[17,18]$. The above results indicate that homogenous nucleation may be taking place at rates previously thought to be consistent only with heterogenous nucleation. It may be that homogenous nucleation plays a much more significant role in polymer foaming that previously thought. To confirm this, more quantitative predictions would be required - see Conclusions and Outlook section 4. In any case, the above results should also modify heterogenous nucleation rate predictions since these rates are often based on modifications of the free energy barrier height used in CNT for homogenous nucleation $[17,18]$.

To understand the deviation of CNT from SCFT for nanocellular polymer systems, one 


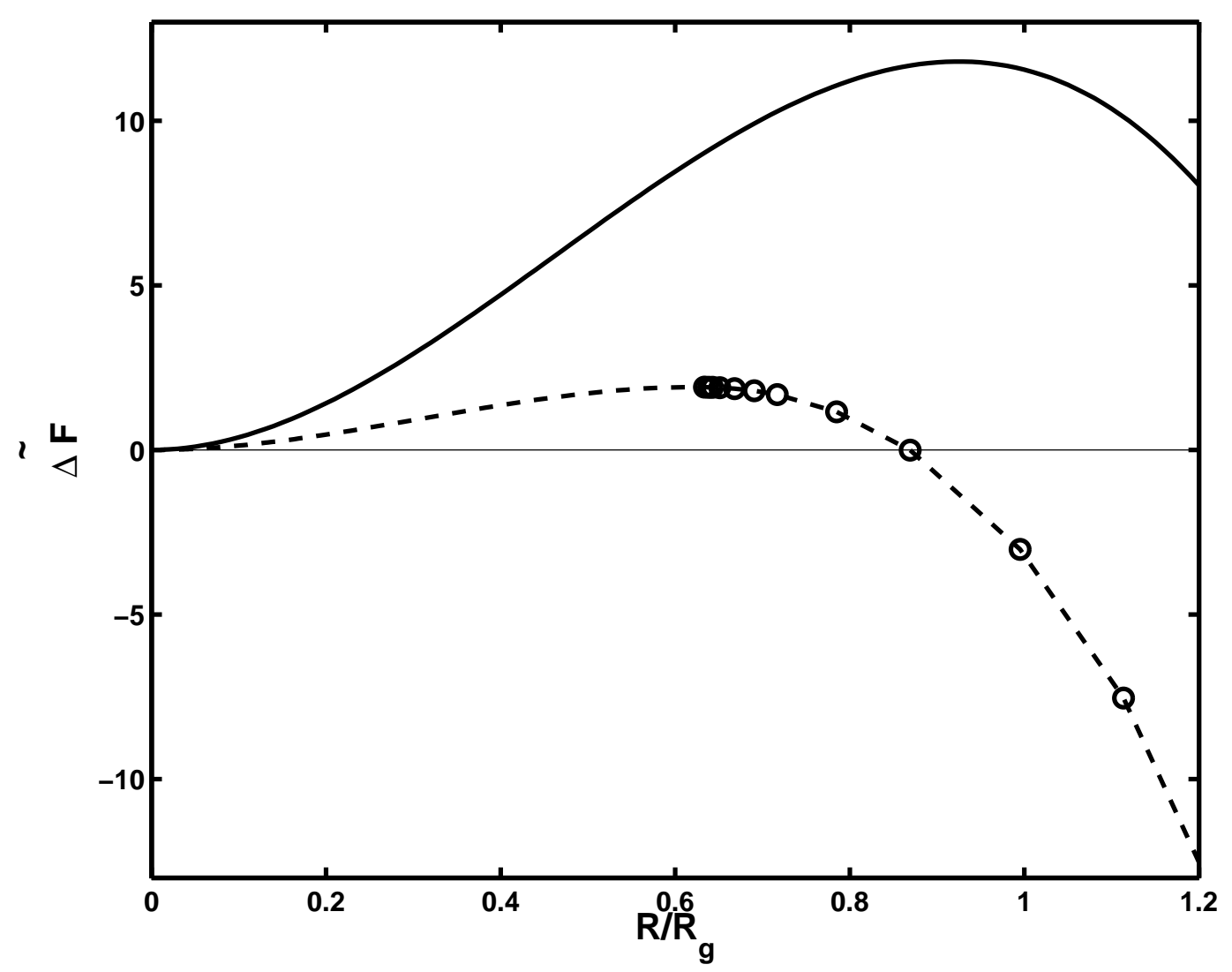

FIG. 3: Free energy of the existence of a bubble, $\widetilde{\Delta F}$, as a function of bubble radius $\tilde{R}$. The solid line is the prediction of classical nucleation theory. Open circles are SCFT predictions with a dashed line guide to the eye.

can notice that with SCFT phrased in the CNT-like form of equation (13), all differences between CNT and SCFT predictions arise due to the bubble radius dependence of the surface tension $\tilde{\gamma}$ and volume free energy density $\widetilde{\Delta \mathcal{F}}_{V}$. We have observed from our calculations that neither $\tilde{\gamma}$ nor $\widetilde{\Delta \mathcal{F}}_{V}$ alone is able to account for a majority of deviation of SCFT from CNT; both quantities are important. We examine $\tilde{\gamma}$ first. The surface tension is plotted as a function of radius $\tilde{R}$ as the thick solid line in figure 4. To understand the microscopic origin of the radius dependence of the surface tension, we have broken it up into thermodynamic components, which we also show in figure 4. Details on this procedure can be found in reference [12]. One observes that it is the configurational entropy contribution to the surface tension (open circle line) and the internal energy contribution (closed dot line) that cause the surface tension to drop with decreasing radius. (Note that all the curves in figure 4 have 


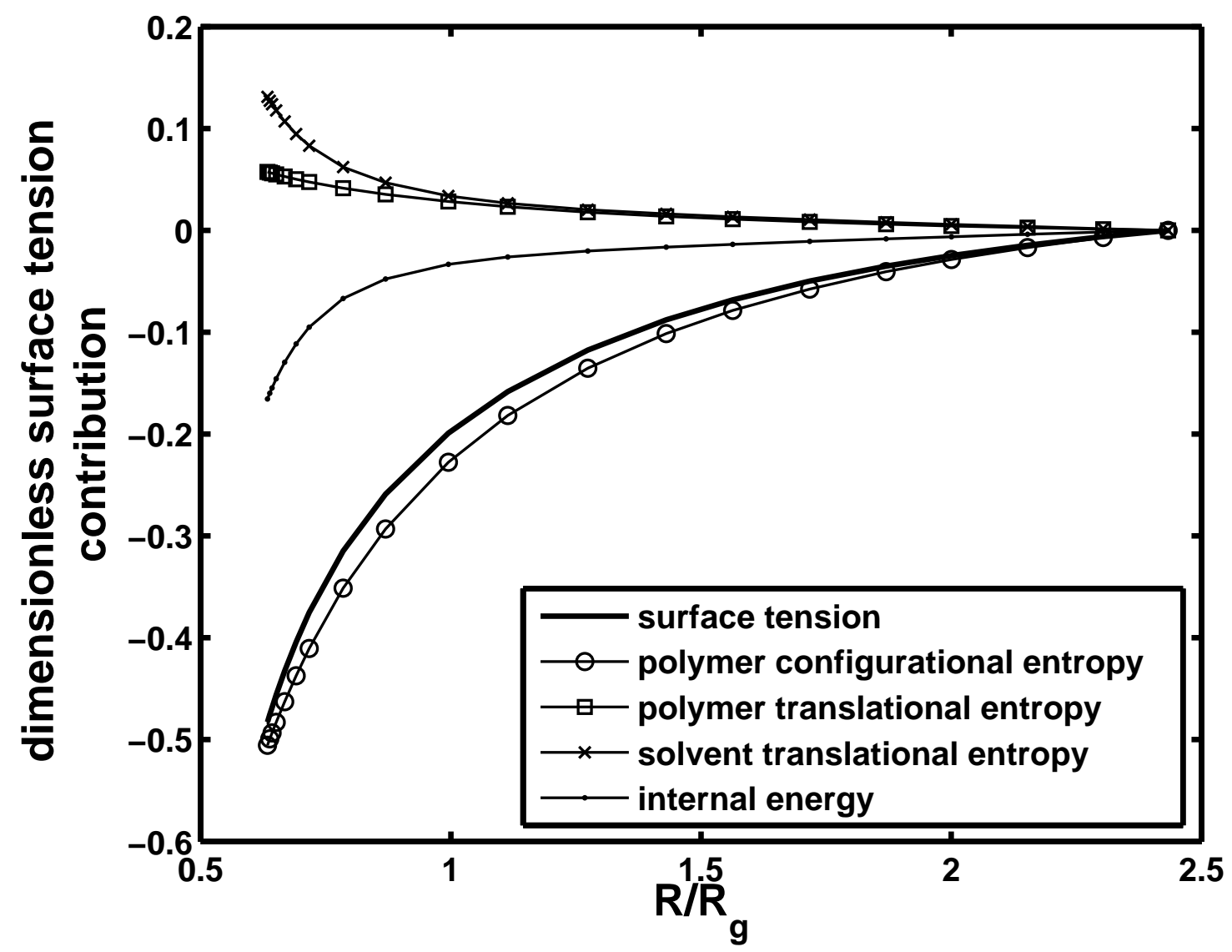

FIG. 4: Contributions to the surface tension of various thermodynamic components as indicated in the legend. For ease of comparison, all components have been shifted by varying amounts to be zeroed at a comparatively large radius of approximately $R=2.5 R_{g}$.

been shifted to a convenient zero in order to easily compare their shapes.) We discuss the conformational entropy contribution first because it is mostly responsible for the drop in surface tension. This is a quite different from our SCFT study of flat surface tensions where the polymer configurational entropy was not a significant factor in predicting behavior [12]. For flat surface tensions then, simple liquid theories can be successful in predicting surface tension behavior as discussed in reference 12 whereas on the nanoscale, simple liquid theories would be inadequate because curvature effects make polymer conformations a dominant factor. This is consistent with experimental findings of Shukla and Koelling for nanocellular foams [3]. They were only able to fit their results with CNT when they modified their surface tension using the Tolman approximation [27]. The Tolman expression is a phenomenological 

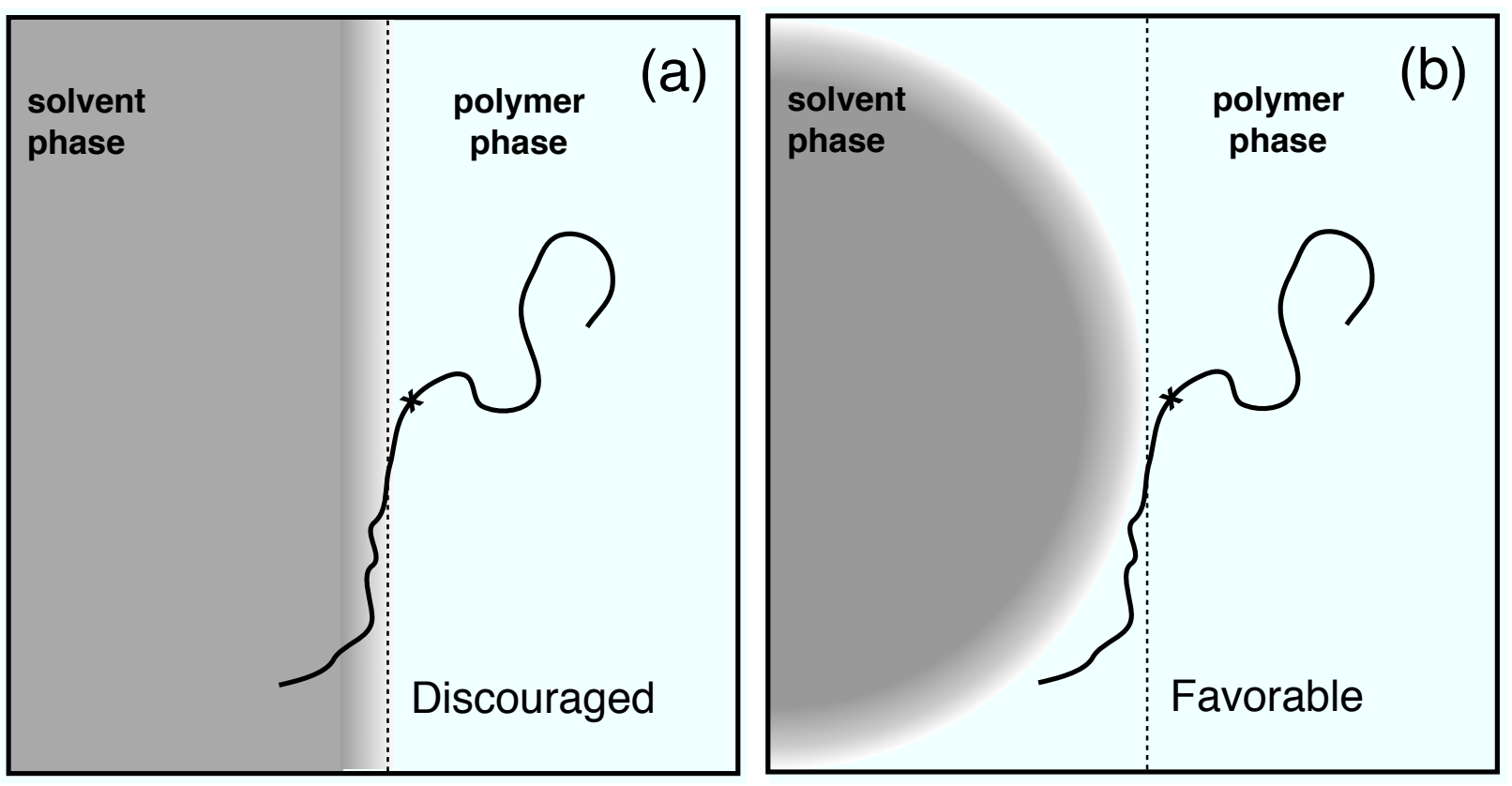

FIG. 5: Schematic showing the differences in conformations and internal energies for polymers located near surfaces. The grey area is the gas bubble region and the white area is the polymer melt region. One polymer is explicitly shown with an " $\mathrm{x}$ " marking its center of mass. (a) A flat surface discourages certain conformations by increasing the internal energy. (b) A curved surface allows these conformations with no additional energy penalty.

expansion that gives surface tension a bubble radius dependence; it has no microscopic information and introduces an arbitrary fitting parameter known as the Tolman length. In contrast, larger bubble foams, including microcellular foams, can sometimes be fitted with CNT, as reported by Goel and Beckman [6]. This brings us to the conformational entropy discussion. For large bubbles, the cell surface appears flat on the length scale of a polymer molecule. The polymer is discouraged from entering the bubble because of an energy penalty for doing so - the gas and polymer molecules are immiscible - and the possible polymer conformations are reduced near the bubble surface - see figure 5(a). As the bubble shrinks to nanometre length scales, the curvature of the surface becomes significant on the length scale of a polymer molecule. As depicted in figure 5(b), regions that were previously less accessible to the polymer are now favorable. The number of polymer conformations per unit bubble surface area are increased relative to a flat surface and the excess free energy of the interface is ultimately lowered. Although the surface area of the bubble is also smaller, which would tend to increase the surface tension, the excess free energy shrinks more rapidly 
so that the surface tension becomes lower than for the flat case. The lower surface tension results in a smaller nucleation barrier and a higher nucleation rate.

The internal energy component of the surface tension is a secondary, less important, contributor to the drop in surface tension with decreasing bubble radius. The same mechanism as for the configurational entropy is found to contribute to the internal energy component drop. Although polymers are discouraged energetically from entering the gas bubble region as depicted in figure 5, some will inevitably venture in. This will occur more frequently for flat surfaces than for curved ones since some conformations that result in bubble entry in the flat case do not enter the bubble in the curved case. This means there will be a lower internal energy per unit bubble surface area for smaller, more curved bubbles, which ultimately results in a lower surface tension. Of course the internal energy per volume increases for smaller bubbles, as observed in figure 2, where we see greater mixing of polymer and gas for the smaller bubble system compared to the larger bubble system. The mechanism described above moderates this increase and so contributes to a dropping surface tension.

From figure 4, one notices that the internal energy surface tension component drops more sharply for radii below about $0.9 R_{g}$. This is due to another factor that starts affecting the structure of the bubble. For these very smallest bubbles, the bubble walls start colliding together, meaning there is no bulk region at all in the interior of the bubble. This increases the mixing of the gas and polymer molecules and raises the internal energy of the system. It turns out that the internal energy of the bulk regions increases more quickly due to this merging of interfaces than the system as a whole, so the system internal energy density minus the bulk internal energy density (the excess internal energy density) drops for the smallest radii instead of increasing. This excess internal energy drop, even though divided by a decreasing bubble surface area, causes the steeper decline of the internal energy component with shrinking radius depicted in figure 4. Overall, the internal energy surface tension component drops both because of the increased curvature of the interface which allows for less mixing, in a relative sense, of gas and polymer near the interface, and, for smaller radii, increased mixing of gas and polymer away from the interface (in the bulk region) when the bubble interior starts to collapse.

The volume free energy density also contributes to the lower nucleation barrier. Figure 6 shows the negative of $\widetilde{\Delta \mathcal{F}}_{V}$ (since the volume term is negative in the CNT formula) as a function of bubble radius together with its thermodynamic components. The negative vol- 


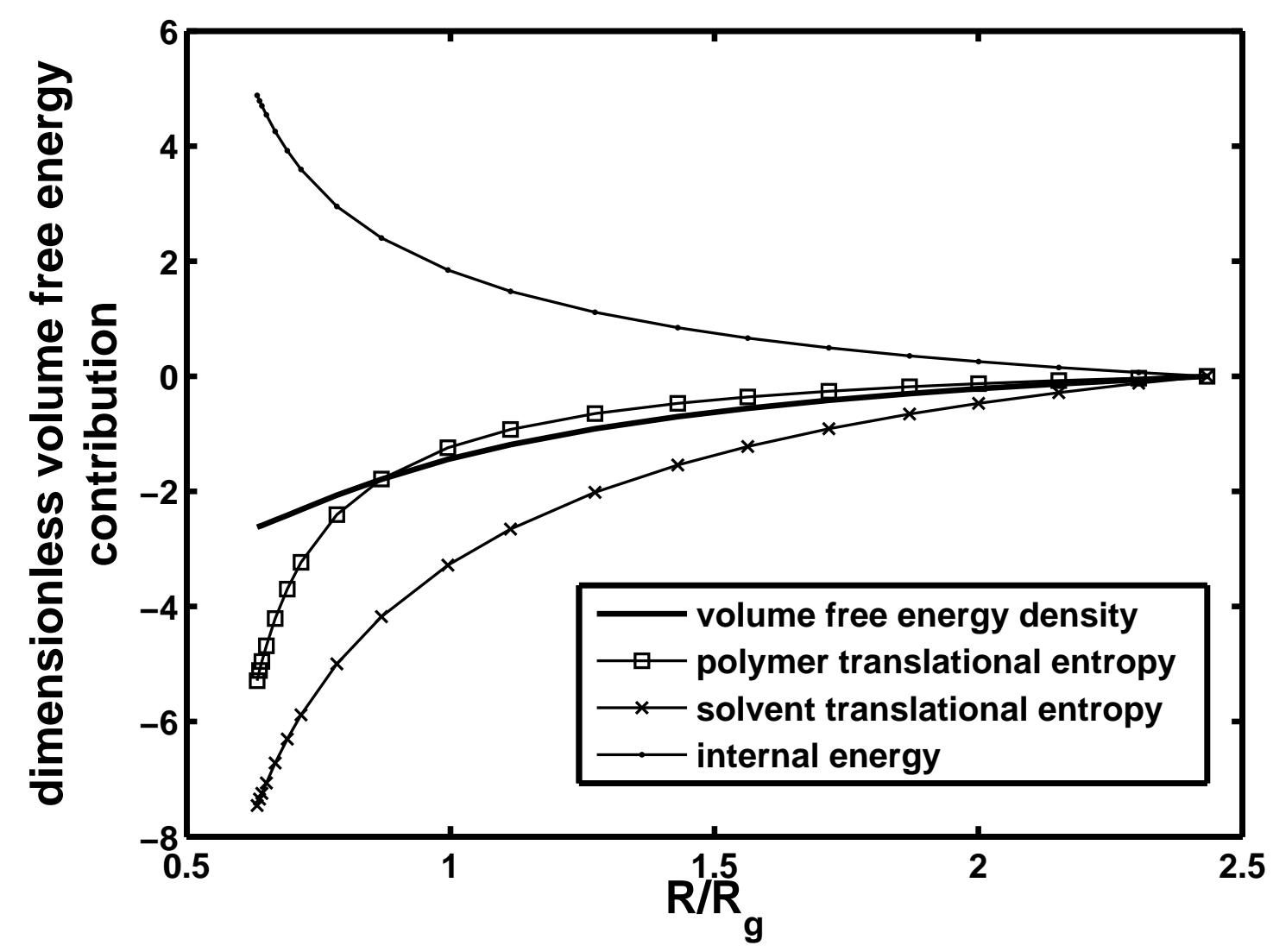

FIG. 6: Contributions to the (negative) volume free energy density of various thermodynamic components as indicated in the legend. For ease of comparison, all components have been shifted by varying amounts to be zeroed at a comparatively large radius of approximately $R=2.5 R_{g}$.

ume free energy density drops (the magnitude of $\widetilde{\Delta \mathcal{F}}_{V}$ increases) with decreasing radius and so contributes to a lower nucleation barrier. From figure 6 we see that the polymer and gas entropic contributions are responsible for this drop. This can be viewed as merely a volume response to the surface mechanisms already described, namely, the increased curvature of the interface and the collapse of the bubble interior which causes increased bulk mixing. From figure 2 we see that a smaller radius bubble has more mixing of polymer and gas than does a larger radius bubble. This means the internal energy per volume rises while the entropic contributions drop (entropies increase) as the radius is decreased. This happens because the extra conformational entropy and internal energy gained with increased interface curvature changes the balance of energy and entropy as does, to a lesser extent, the extra bulk mixing when the bubble walls collide. 
The internal energy contribution to the surface tension drop, due to both the curvature effect and the increased deviation from bulk conditions resulting from a collapsing bubble, was usually found to be a less important contributor to the failure of CNT than the polymer configurational entropy curvature effect. The greatest internal energy contribution to the surface tension drop was found for $\chi N=160, \phi_{s}=0.26$ and $\alpha=0.01$, where we found this effect approached the magnitude of the configurational entropy effect. One might suppose that for higher segregations still, the internal energy contribution might become the dominant factor. Real polymer foam systems would be expected to have lower values of $\alpha$ than used here and higher $\chi N$ values, so the internal energy mechanisms, especially due to a collapsing bubble, should not be discounted until further numerical studies are possible. Computational limitations prevent us from exploring such cases in this work. Previous work by Lee and Flumerfelt predicted that increased mixing of polymer and gas would be the primary cause of the failure of CNT for polymer foaming systems as opposed to curvature effects [15]. However they weren't able to examine the microscopic origins of these factors since they assumed a perfectly sharp bubble interface and didn't use a model containing polymer degrees of freedom. Thus the mixing mechanism was, by construction, the only candidate.

It is interesting to note that comparisons of CNT to other, non-polymeric, systems, show that CNT fails for reasons related to the present system. There have been many recent expositions of the failure of CNT, for example, references 28 (and references therein), 29-33, that show CNT fails for small bubble or droplet sizes. In particular, density functional studies reveal that it is the long range attractive portion of the particle interactions that are responsible for the failure of CNT [19], 31 (and references therein). This is analogous to the present conformational entropy effect in polymers since this non-local nature of macromolecules represents an effective longer range potential.

\section{CONCLUSIONS AND OUTLOOK}

We have compared classical nucleation theory to self-consistent field theory using an identical model. For nanoscale bubbles, where the curvature of the bubble surface is comparable to polymer molecular sizes, we found, in the reported example, that classical nucleation theory is more than a third too large in its estimation of the critical radius, more than six 
times too large in its estimation of the nucleation barrier and more than five orders of magnitude too small in its estimation of the nucleation rate. We found similar disagreements for other parameter values. Given that polymer foaming is thought to be dominated by heterogenous nucleation $[17,18]$ in part because CNT predicts a negligible rate for homogenous nucleation, our SCFT results provide circumstantial evidence that there may be much more homogenous nucleation taking place in polymers foams than previously thought. Our results also suggest that heterogenous nucleation rates will be different than predicted by some models $[17,18]$.

The main microscopic origin of the CNT error was the increased number of conformations available to polymer molecules in the vicinity of a curved interface compared to a flat interface. Related to this, mixing of polymer and fluid was also reduced, in a relative sense, in the vicinity of a curved interface compared to a flat interface. Another mechanism for the failure of CNT, found for very small bubbles, was increased bulk mixing due to the disappearance of the bulk region in the interior of the bubble. While this effect is less important than the curvature effect, different, experimentally appropriate, parameter choices could change the relative importance. Although such parameter choices are computationally inaccessible to us at present, choosing polymers that have a volume very much greater than fluid molecules would justify the use of the ground state approximation $[9,10]$. This could both simplify the SCFT formalism and, perhaps, make accessible other choices of system parameters.

For quantitative predictions, not only should longer polymers be used, but equation of state effects should be included. Using equation of state effects in polymer SCFT surface tension calculations has been discussed elsewhere [11,12]. These equations of state methods could be combined with the ground state approximation to allow for quantitative but simple models of nanocellular polymer foam nucleation. In particular, the Simha-Somcynsky equation of state has been shown to be very effective for describing the behaviors of polymer$\mathrm{CO}_{2}$ mixtures [34]. Heterogenous nucleation could also be studied using SCFT techniques borrowed from the study of nanocomposite materials [35]. 


\section{Acknowledgments}

The authors acknowledge financial support from NSERC Canada in the form of a Strate-

gic Projects Grant and Discovery Grants. RBT acknowledges helpful discussions with M. W. Matsen and V. V. Ginzburg.

[1] S.-T. Lee, C. B. Park, and N. S. Ramesh, Polymeric Foams: Science and Technology (CRC Press, Boca Raton, 2007).

[2] S. N. Leung, A. Wong, Q. Guo, C. B. Park, and J. H. Zong, Chem. Eng. Sci. 64, 4899 (2009).

[3] S. Shukla and K. W. Koelling, Ind. Eng. Chem Res. 48, 7603 (2009).

[4] S. Siripurapu, J. A. Coughlan, R. J. Spontak, and S. A. Khan, Macromolecules 37, 9872 (2004).

[5] S. K. Goel and E. J. Beckman, in Cellular Polymers 2nd International Conference (Rapra Technology Limited, Heriot-Watt University, Edinburgh, U. K., 1993), pp. 251-274.

[6] S. K. Goel and E. J. Beckman, Polym. Eng. Sci. 34, 1137 (1994).

[7] D. W. Oxtoby, Annu. Rev. Mater. Res. 32, 39 (2002).

[8] A. E. Kuchma, F. M. Kuni, and A. K. Shchekin, Phys. Rev. E 80, 061125 (2009).

[9] G. H. Fredrickson, The Equilibrium Theory of Inhomogeneous Polymers (Oxford University Press, New York, 2006).

[10] M. W. Matsen, in Soft Matter Volume 1, edited by G. Gompper and M. Schick (Wiley-VCH, 2005), pp. 87-178.

[11] K. Binder, M. Müller, P. Virnau, and L. G. MacDowell, Adv. Polym. Sci. 173, 1 (2005).

[12] H. Park, R. B. Thompson, N. Lanson, C. Tzoganakis, C. B. Park, and P. Chen, J. Phys. Chem. B 111, 3859 (2007).

[13] R. B. Thompson, J. R. MacDonald, and P. Chen, Phys. Rev. E 78, 030801 (2008).

[14] R. B. Thompson, C. B. Park, and P. Chen, J. Chem. Phys 133, 144913 (2010).

[15] J. G. Lee and R. W. Flumerfelt, J. Colloid Interf. Sci. 184, 335348 (1996).

[16] K. Y. Kim, S. L. Kang, and H.-Y. Kwak, Polym. Eng. Sci. 44, 1890 (2004).

[17] S. N. Leung, C. B. Park, and H. B. Li, Plast. Rubber Compos. 35, 93 (2006).

[18] S. N. Leung, A. Wong, C. B. Park, and J. H. Zong, J. Appl. Polym. Sci. 108, 39974003 (2008). 
[19] D. W. Oxtoby and R. Evans, J. Chem. Phys. 89, 7521 (1988).

[20] R. A. L. Jones and R. W. Richards, Polymers at Surfaces and Interfaces (Cambridge University Press, Cambridge, 1999).

[21] The surface tension formulas (14) of reference 12 and (2) of reference 13 are missing a factor of 6 on the left hand side. This has no impact on any results.

[22] D. Duque, in his work on block copolymer micelles, describes a different SCFT approach that could be used to calculate the free energy of a bubble that doesn't require changing the system volume [26]. This grand canonical method would hold the exterior bulk volume fractions constant at the experimental conditions. This would give a free energy barrier for a completely isolated bubble and would be used to predict very early time nucleation rates. We feel our method is a more realistic approach for the case of polymer foams where a majority of nucleation events may take place in an environment of other bubbles. It should also be more adaptable to heterogenous nucleation.

[23] M. W. Matsen and F. S. Bates, J. Chem. Phys 106, 2436 (1997).

[24] S. N. Leung, H. Li, and C. B. Park, J. Appl. Polym. Sci. 104, 902 (2007).

[25] S. M. Wood and Z.-G. Wang, J. Chem. Phys 116, 2289 (2002).

[26] D. Duque, J. Chem. Phys 119, 5701 (2003).

[27] R. C. Tolman, J. Chem. Phys 17, 333 (1949).

[28] I. J. Ford, Proc. Instn. Mech. Engrs. Part C: J. Mechanical Engineering Science 218, 883 (2004).

[29] A. V. Neimark and A. Vishnyakov, J. Chem. Phys. 122, 174508 (2005).

[30] J. Merikanto, E. Zapadinsky, A. Lauri, and H. Vehkamäki, Phys. Rev. Lett. 98, 145702 (2007).

[31] I. E. Parra and J. C. Gran̆a, J. Chem. Phys 132, 034702 (2010).

[32] I. Napari, J. Julin, and H. Vehkamäki, J. Chem. Phys 133, 154503 (2010).

[33] S. Ghosh and S. K. Ghosh, J. Chem. Phys 134, 024502 (2011).

[34] M. M. Hasan, Y. G. Li, G. Li, C. B. Park, and P. Chen, J. Chem. Eng. Data 55, 48854895 (2010).

[35] M. W. Matsen and R. B. Thompson, Macromolecules 41, 1853 (2008). 